\title{
ÉLÉMENTS POUR UNE TYPOLOGISATION DE LA NARRATION RADIOPHONIQUE
}

\author{
Frédéric Antoine ${ }^{1}$
}

Le récit faisait originellement intrinsèquement partie d'un des fondements du langage et de l'offre programmatique radiophonique. La concurrence intermédiatique évinça par la suite une large part du champ narratif du domaine de la radio. Depuis quelques années, on assiste toutefois à un réinvestissement du média par des formes narratives fort diverses. Ayant observé cette évolution et déterminé les invariants organisant les rapports entre le média et l'univers du récit, cet article entend aider à l'analyse actuelle de ce domaine en proposant de typologiser ces récits narratifs et d'envisager leur approche dans des matrices analytiques.

1 Frédéric Antoine est professeur à l'Université catholique de Louvain et membre de l'ORM.

Recherches en communication, $\mathrm{n}^{\circ} 37$ (2012). 
Innombrables sont les récits du monde. [...] Le récit peut être supporté par le langage articulé, oral ou écrit, par l'image, fixe ou mobile, par le geste et par le mélange ordonné de toutes ces substances; il est présent dans le mythe, la légende, la fable, le conte, la nouvelle, l'épopée, l'histoire, la tragédie, le drame, la comédie, la pantomime, le tableau peint [...], le vitrail, le cinéma, les comics, le fait divers, la conversation.

Comme l'affirmait déjà Roland Barthes dans l'introduction de son texte fondateur sur l'analyse structurale du récit (Barthes, 1981, p. 7), la forme narrative " commence avec l'histoire même de l'humanité », a touché tous les peuples et n'a depuis cessé de se développer. Par l'intermédiaire des médias, il s'est aujourd'hui fait omniprésent, ainsi que le rappelle Mars Lits :

Nous n'accédons au monde que par l'intermédiaire du langage, et l'utilisation de ce langage permet d'organiser des séquences narratives plus ou moins structurées selon des règles précises. Si l'organisation superficielle, l'apparence du discours, peut être conditionnée par le canal utilisé, il y a cependant des invariants narratifs constitutifs de tout récit. (Lits, 2008, p. 5)

\section{Radio et médiagénie}

S'il est acquis, notamment depuis les travaux de l'Observatoire du Récit Médiatique de l'Université catholique de Louvain (Antoine, 1997), que les récits jouent un rôle déterminant dans la construction des identités, et que cette fonction est actuellement remplie de manière essentielle par les médias, il n'en reste pas moins que le mode d'expression narratif n'a pas investi avec la même vigueur l'ensemble des moyens de communication actuels. Chaque média dispose en effet d'une " médiagénie propre » (Marion, 2007, p. 85) et est plus ou moins prédestiné à véhiculer avec aisance un contenu narratif. Il va même de soi que certains supports de communication, en particulier de nature iconique, entretiennent une relation quasiment fusionnelle avec la notion de récit, liée à la " narrativité inhérente » des images qui en constituent l'essentiel (Brau \& Lavergne, 1991).

Sans reformuler ici l'ensemble de l'histoire des relations entre les diverses formes médiatiques et le narratif depuis la nuit des temps, on reconnaîtra que le même caractère inhérent n'est pas l'apanage d'autres 
supports de communication, notamment suite aux spécificités des opérations de formalisation de l'expression narrative qu'ils impliquent.

La radio est de ceux-là. Média de la parole, elle relève plus naturellement de l'ordre du discours que de celui du récit, ainsi que l'affirmait Patrick Charaudeau dans le premier numéro de la collection «Langages, discours et sociétés »:

Vecteur de la parole, le média radio s'est naturellement inscrit dans la sphère du discursif et elle s'y est en grande partie maintenue. (Charaudeau, 1984)

De nos jours, la radio n'apparait-elle pas d'abord comme le lieu de l'énonciation, de l'annonce de la nouvelle, de la désannonce d'une programmation musicale ? Et, à jeter un coup d'œil dans le rétroviseur radiophonique, ne se remémore-t-on pas en premier lieu de grands moments liés à la fonction discursive du média, support dès sa naissance de bon nombre de conférences et « causeries radiophoniques », à commencer par les « fireside chats» du président F. Roosevelt, promotionnant de la sorte par la radio sa politique de New Deal dès 1933.

Cette assimilation de la radio à un média d'expression discursive y a entraîné une survalorisation du rôle de l'ego-énonciateur, que celuici y remplisse la fonction de "speaker », animateur, présentateur ou journaliste. Mais les confiner à ce rôle serait oublier que ces personnages du système radiophonique y ont aussi fait fonction d'énonciateurs narratifs.

\section{Radio et narration : l'Âge d'or}

Car, très rapidement après son avènement, la radio n'a pu résister aux tentations de la narrativité. Étape par étape, la narration est entrée dans le paysage radiophonique et s'y est installée au point d'y devenir une évidence. Dans certains de ses registres, elle est alors passée du discours à la narration en recourant à l'exploitation de la richesse de l'expression vocale, des sons et des formes musicales.

Ce que l'on a surnommé «l'Âge d'or » de la radio s'est caractérisé par le rôle qu'y a joué le narratif. 
The so-called Golden Age of radio began in 1930 when Americans huddled by their sets in the evening for entertainment. Listening to live radio broadcasts was the new and exciting entertainment form of the day. (Osenton, 2002)

Ainsi, alors que la part discursive du radiophonique s'était développée en rapport avec l'univers du réel, le narratif y crût lorsque le ludique s'empara de ce média, en faisant un élément incontournable des palettes du divertissement.

The latter years of the 1930s represented a golden age for radio. Radio became increasingly accepted as an essential part of American life and, before competition from television materialized, it was the supreme form of home entertainment, offering a wide range of programs. (Cashman, 1989)

En peu de temps, les récits radiophoniques, présentés sous forme de dramatiques ou de feuilletons, prirent une place prépondérante dans la programmation non musicale des stations. Une analyse de l'offre des radios affiliées au réseau CBS en avril 1934 relève que « script, including drama and dialogue, was broadcast more than any other nonmusical type of program » (Allport \& Cantril, 1935). À cette époque, par le truchement de la diffusion de fictions, la narration s'inscrit au cœur du radiophonique. Toute étude sur la place de la fiction dans l'écriture radiophonique ne peut éviter de faire référence à la Guerre des Mondes (War of the Worlds) produite par Orson Welles depuis le Mercury Theatre on The Air de New York le 30 octobre 1938 (Flynn, 2005). Mais ce serait oublier que les premières dramatiques radiophoniques et les premiers " radios soaps » furent diffusés en soirée aux USA dès le début des années 1920, et que les feuilletons (« daily soaps ») apparurent sur les ondes américaines à l'orée des années 1930.

En France, la première diffusion de fiction radiophonique est considérée comme ayant eu lieu sur Radiola en novembre 1922 (Baudou, 1995). Et dès 1924, un concours est organisé à Paris afin de promouvoir ce genre radiophonique, Gabriel Germinet créant alors une panique proche de celle que Welles suscitera plus tard lors de la diffusion de son œuvre Maremoto (Meadel, 1991). Les feuilletons courts s'inspireront pour leur part de La famille Duraton, dont la diffusion débute sur Radio-Cité Paris dès 1937. Il deviendra un classique de ce type de programmes après guerre sur Radio Luxembourg (Prot, 2007). Dans 
l'orbite francophone européenne, l'Âge d'or des dramatiques radiophoniques se situera au cours des années 1950.

\section{Invariants et évolutions}

Tim Crook relève ce qui constitue alors la liste des « composants de base » de feuilleton radiophonique : The Beginning, The Moment of Arrival, Structure, The Plot, Surprise, Character, Conflict, Polarities or Extremes, The Climax, Dialogue, Atmosphere / Ambience, Emotion, Balance Character and Plot, Purpose, Tension and Humor (Crook, 1999). Crook y ajoute plusieurs « principes de base » relevés lors de l'analyse de "radio dramas » : "The principle of developing scenes », « The principle of character » et « The principle of hero/heroine».

S'ils figurent en bonne place parmi les ingrédients assurant le succès des fictions radiophoniques, les invariants identifiés par Crook ne s'appliquent pas à ce seul genre de programme. On peut considérer que toute narration radiophonique repose sur une alchimie où se mélange la plupart de ces différents éléments, voire qu'ils peuvent aussi s'appliquer à une vaste panoplie de récits, même en dehors de l'univers de la radio.

Comme l'ont mis en exergue tous les analystes du récit, aucune narration ne peut ainsi exister sans personnages, voire sans héros, ni sans intrigue («plot») ou structure. Bon nombre de productions narratives, et en particulier dans un cadre d'exploitation sonore, requièrent la présence de dialogues, la création d'atmosphères (ambiance) et l'expression d'émotions. Autant d'éléments qui font de ces productions un « genre » particulier, dont Crook regrette qu'il n'ait pas davantage jusqu'ici attiré l'attention des analystes et des chercheurs.

Radio drama has probably been the most unappreciated and understated literary form of the twentieth century and I hope the neglect will not continue into the twenty first century. Academics, media theorists and writers in most cultures have in the main failed to realise that the medium of sound has provided an environment in which a new storytelling genre has been born. It has developed with sophistication and explosive energy, and now occupies a significant position in the cultural lives of societies throughout the globe. Even where the dominance of long-form popular dramas has transferred to television, the audio drama 
narrative is central to the short narrative communication of radio commercials. Huge traditions, styles and movements have been established and remain largely undocumented. Even now, radio drama is regarded as an adjunct of radio production practice. The shelves of the library of Goldsmiths College, University of London disclose the following ratio of critical publication space between novels and poetry, theatre texts, film/television and radio drama : 64, 21, 14, 1 ! (Crook, 1999)

Comme le note Crook, avec l'avènement de la télévision, le narratif semble avoir quitté le radiophonique, ou se contente d'y subsister dans quelques types de programmes de niches, comme nous en avions personnellement fait le constat dès le début des années 2000 (Antoine 2006a). Le développement de médias narrativement plus « narratogéniques " avait ramené la radio à sa condition première de lieu d'expression de la communication discursive. Mais en parallèle, à la même époque, nous avions déjà pu observer le début d'un certain retour de la présence du narratif dans le média radio, notamment dans le domaine des spots publicitaires (Antoine 2006b). Comme si, à l'instar de ce qui s'était passé à son Âge d'or, le récit cherchait à nouveau " enchanter » la radio.

La diversification progressive del'offre en stations et en programmes qui fit suite à la vague de dérégulation des systèmes audio(visuels) dans les années 1980 et 1990 n'est sans doute pas indirectement étrangère au phénomène. Mais d'autres évolutions du paysage médiatique et de la société dans son ensemble ont aussi concouru à fournir des éléments tendant à expliquer ce reversement de tendance. L'évolution technologique, qui a permis à la radio de s'extraire du carcan de la transmission hertzienne, commence à offrir à l'expression radiophonique de nouveaux champs de développement au sein desquels le récit pourrait trouver sa place (Antoine, 2009). Mais la raison la plus profonde de ce nouvel intérêt de la radio pour le narratif paraît résider ailleurs, et être associé au phénomène d'essaimage du modèle narratif auquel on assiste actuellement dans tous les domaines de la culture, notamment sous l'influence de l'extension des techniques de «storytelling ». Depuis les années 1990, le récit semble en effet s'imposer comme le meilleur moyen pour communiquer avec les opinions publiques, et les audiences paraissent elles-mêmes en demander toujours davantage. Dans pareil contexte, la radio pouvait-elle rester à l'écart d'un mode d'expression 
par ailleurs si parfaitement installé, au risque de voir confortée dans son image de média de masse d'une ère révolue?

\section{Essai typologique de narrativité radiophonique}

Ce réinvestissement du narratif dans le radiophonique devenant de plus en plus tangible, il devrait, à l'avenir, pouvoir être pris en considération au sein d'une matrice typologique pour laquelle nous proposons ici quelques axes fondamentaux, basés sur les modes classificatoires des programmes radiophoniques (et télévisuels) dénommés ESCORT mis en place par l'UER (Union Européenne de Radiodiffusion) (EBU, 2007).

Pour rappel, la classification UER « identifies a common set of programme and service concepts and genres, which form part of the descriptive information of a service (e.g. news) or of a programme (e.g. comedy) ». La classification est « a hierarchical scheme organised in a series of dimensions describing material in a rising level of detail » selon sept dimensions : "Intention, Format, Content Participation, Intended Audience/Target Group, Origination, Content Alert ». Dans le cadre qui nous occupe ici, nous recourrons aux premières dimensions proposées, à la fois afin d'établir les axes de notre matrice et pour en définir les éléments constitutifs. Dans le but d'inscrire ces éléments en regard de la question de la narrativité radiophonique, nous y ajouterons des variables propres à l'analyse des récits.

Sur cette base, nous proposons d'inscrire les programmes radiophoniques, tels qu'ils sont désignés en langue anglaise dans la classification UER adaptée pour la circonstance à la langue française, dans un espace organisé selon deux axes. Cette disposition permet à la fois d'identifier les programmes en fonction des deux critères et de les positionner relativement les uns par rapport aux autres selon l'intensité avec laquelle ces deux indicateurs opèrent sur chaque production.

Le premier axe, que nous avons ici inscrit en horizontalité, distingue les narrations relevant de la sphère du réel de celles dépendant de la sphère du fictionnel. Le second distribue les programmes en vertu de leur intensité narrative, créant une discrimination dans le statut à ce propos selon que cette narrativité soit intrinsèque au programme, c'està-dire inhérente à l'essence et à la nature de son contenu ou selon que la narrativité en soit extrinsèque, c'est-à-dire liée à sa mise en forme, 
et donc à son organisation (ou sa transformation) par une instance de production.

En vertu de cette distribution, en sélectionnant dans les inventaires typologiques de programmes empruntés à la classification UER les émissions à contenu ou forme narrativisante, nous pouvons obtenir une répartition graphiquement représentable comme suit :

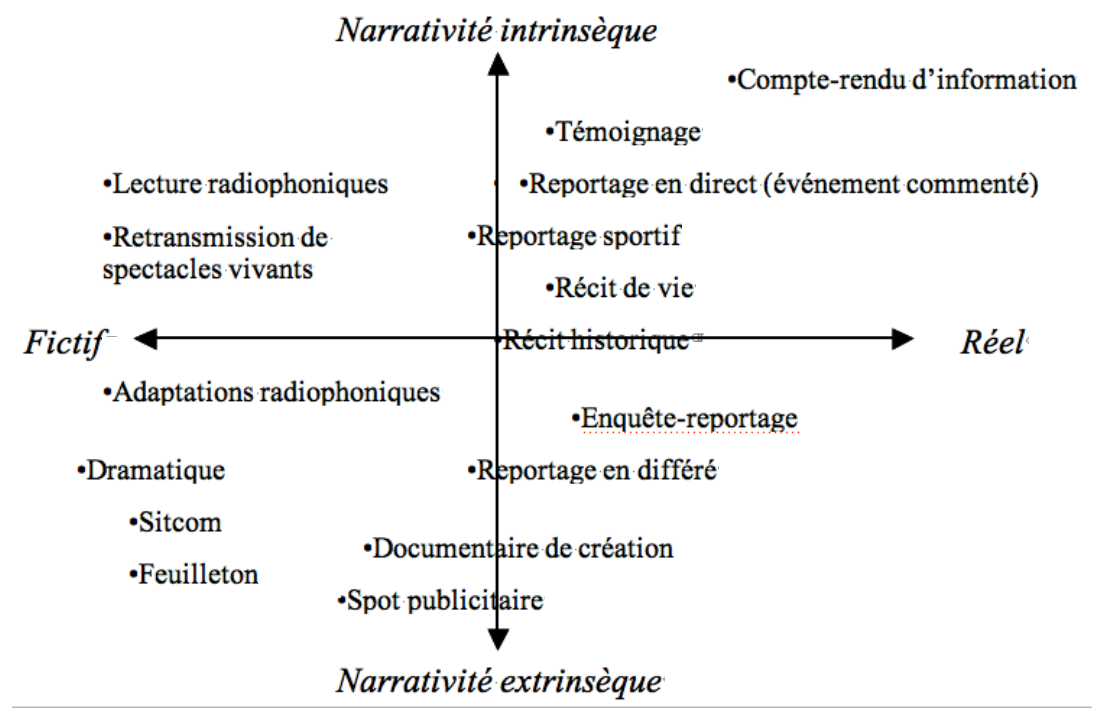

L'inventaire des contenus de cette topographie axiale peut s'opérer par analyse des composantes des quatre parties qui la constituent. L'organisation de ce mapping n'est évidemment pas exempte d'une certaine subjectivité. Il y a donc lieu de considérer qu'il s'agit de proposer ici une première approche de ce que pourrait être une distribution catégorielle de l'offre narrative radiophonique, à peaufiner au fur et à mesure que des travaux plus précis se seront penchés sur ses différentes composantes. Précisons enfin, si besoin en est, que ce projet de mapping ne prend bien sûr pas en compte l'entièreté de l'offre radiophonique mais se concentre sur les programmes présentant une part potentielle appréciable de narratif.

Le cadran supérieur droit rassemble les programmes relatifs à la sphère du réel possédant une narrativité intrinsèque, ordonnés en fonction de l'intensité de leur rapport à la réalité et de la part de 
reconstruction narrative qu'ils contiennent. On y trouvera la diffusion de nouvelles, dénommés faute de mieux « compte-rendu d'information », mais dont les termes anglais « News with story » ou « News report » représentent peut-être mieux l'intitulé. Un peu plus bas sur l'échelle des deux axes figurent les témoignages recueillis et diffusés («Testimonies »), les reportages d'événements se déroulant en temps réel (« Live reports ») et les récits de vie recueillis et diffusés (« Life stories »). Le récit historique a été inscrit côté « réel » à mi-chemin sur l'axe narrativité intrinsèque/extrinsèque, car il relève des deux modèles. Quant au reportage sportif (« Sports live reports »), nous considérons qu'il présente un positionnement à peu près semblable, et pour des raisons identiques, sur l'axe fictif/réel.

Le cadran inférieur droit du tableau situe les programmes relevant de l'univers du réel, mais dont la narrativité est plutôt extrinsèque, c'està-dire liée à la forme conférée au contenu par l'écriture radiophonique et le processus de mise en ondes. C'est là que figure le domaine classique des émissions d' " enquêtes-reportages » et en particulier celui des enquêtes journalistiques ("Investigation reports »).

Tout comme, dans le cadran supérieur, nous avions légèrement décalé du côté " fiction » la catégorie consacrée à la retransmission commentée d'événements sportifs en direct, nous avons de même choisi de positionner un peu plus vers la gauche les productions reposant davantage sur le reportage en différé, plus judicieusement dénommé en anglais « Feature report», dont le travail d'écriture contribue non seulement à développer la narrativité mais aussi la relativité de la distance avec la réalité vécue.

Nous développerons la même appréciation à propos du « documentaire de création », que nous situons pour des raisons identiques dans le cadran inférieur gauche de notre modèle, mais en soulignant qu'il ne présente qu'une faible intensité fictionnelle. Tel n'est évidemment pas le cas des productions radiophoniques reposant sur la mise en ondes d'œuvres naturellement fictionnelles comme les dramatiques, séries ou feuilletons ( tations radiophoniques d'œuvres littéraires.

Reste enfin le cas des spots publicitaires, qui n'appartient pas aux mêmes catégories de programmes que les productions susmentionnées, mais qui se situent également dans ce même cadran inférieur droit, tant en raison de leur intense narrativité extrinsèque qu'en fonction de leur part de caractère fictionnel. 
Le dernier cadran, enfin, héberge les programmes dont la narrativité ne relève pas de leur mise en ondes, mais qui relèvent du monde du fictionnel. On trouvera ici les lectures d'œuvres («Reading ») ou les captations de spectacles vivants («Live performance »).

\section{Matrices narratives}

Un simple regard à l'inventaire exposé dans la matrice permet au lecteur de constater que, même si ces identifications de programmes figurent bien dans des classifications exhaustives toujours de mise à l'heure actuelle, certaines d'entre elles n'apparaissent en réalité que de manière infime, dans les grilles de programmation des stations de radio. Ainsi en est-il d'une large part des programmes identifiés comme se situant dans la partie la plus extérieure de l'axe fictionnel. Toutefois, c'est peut-être de ce côté que la renaissance de productions et d'une écriture radiophonique originale est actuellement en développement. La partie droite du mapping, en relation avec l'axe du réel, paraîtra par contre plus traditionnellement inscrite dans l'univers radiophonique classique.

Par ailleurs, au-delà de l'inscription des programmes identifiés selon les axes que nous avons définis, il doit y avoir lieu de mener une étude plus approfondie des composantes narratives de chacun des axes et des genres ainsi identifiés. Nous proposons à ce propos de recourir à l'inventaire des composants fondamentaux du récit radiophonique présentés précédemment dans ce texte. Il deviendrait ainsi possible de déterminer les invariants caractérisant la spécificité des productions de récits radiophoniques actuels.

En ce qui concerne les axes que nous avons établis, ceux-ci pourraient être organisés selon la matrice suivante :

\begin{tabular}{|c|c|c|c|c|c|c|c|}
\hline & Characters & Heroes & Dialogue & Structure & Plot & Emotion & Ambience \\
\hline \multicolumn{8}{|c|}{ Inner narrativity } \\
\hline \multicolumn{8}{|c|}{ External narrativity } \\
\hline \multicolumn{8}{|l|}{ Fiction story } \\
\hline Real life story & & & & & & & \\
\hline
\end{tabular}


Le même système matriciel pourrait être développé de la sorte pour chaque type de programmes radiophoniques identifié comme potentiellement narratif :

\begin{tabular}{|c|c|c|c|c|c|c|c|}
\hline & Characters & Heroes & Dialogue & Structure & Plot & Emotion & Ambience \\
\hline \multicolumn{8}{|l|}{ Documentary } \\
\hline \multicolumn{8}{|l|}{ Drama } \\
\hline \multicolumn{8}{|l|}{ Feature report } \\
\hline \multicolumn{8}{|c|}{ Historical programs } \\
\hline \multicolumn{8}{|l|}{ Investigation report } \\
\hline \multicolumn{8}{|l|}{ Life stories } \\
\hline \multicolumn{8}{|l|}{ Live performance } \\
\hline \multicolumn{8}{|l|}{ Live report } \\
\hline \multicolumn{8}{|l|}{ Narrative testimony } \\
\hline \multicolumn{8}{|l|}{ News includ. story } \\
\hline \multicolumn{8}{|l|}{ News report } \\
\hline \multicolumn{8}{|l|}{ Radio Ad } \\
\hline \multicolumn{8}{|l|}{ Radio adaptation } \\
\hline \multicolumn{8}{|l|}{ Reading } \\
\hline \multicolumn{8}{|l|}{ Sitcom } \\
\hline \multicolumn{8}{|l|}{ Soap } \\
\hline Sport live report & & & & & & & \\
\hline
\end{tabular}

Que ce soit au niveau de la distribution selon les axes ou de celui des composantes des matrices présentées ci-dessus, il y aurait maintenant lieu d'étudier la manière dont cette topographie résonne dans divers contextes expérimentaux : s'applique-t-elle de manière identique à divers types de radios (privées $\mathrm{v} / \mathrm{s}$ publiques, généralistes $\mathrm{v} / \mathrm{s}$ thématiques, locales $\mathrm{v} / \mathrm{s}$ nationales, public large $\mathrm{v} / \mathrm{s}$ public ciblé...) ? Le modèle est-il européo-centré, voire influencé par la culture francophone de son auteur? Fonctionne-t-il de manière comparable dans des contextes culturels différents, ou divers créneaux horaires ? Des études longitudinales permettraient également d'observer les variations de l'offre narrative selon les périodes (années, saisons...) 
À partir des éléments posés jaillit une variété de questions de recherches qui devrait permettre d'appréhender l'offre radiophonique sous un jour nouveau. Elles devraient offrir l'opportunité de valider ou d'infirmer, au cas par cas, les observations et analyses générales exprimées dans cet article, qui s'est essentiellement voulu conçu comme un aiguillon.

\section{Références}

Allport, G.W., \& Cantril, H. (1935). The psychology of radio. New York : Harper.

Antoine, F. (1997). Le récit médiatique : l'objet et sa méthode. Pour une mise en tension dynamique. Recherches en communication, 7, pp. 173-179.

Antoine, F. (2006a). La mise en récit et le futur de la radio. communication au colloque La radiodiffusion au tournant du siècle, GRER-Université Lyon 3.

Antoine, F. (2006b). Radio et récits : du cas emblématique des spots publicitaires à une Renaissance. Recherches en communication, 26, pp. 33-44.

Antoine, F. (2009). Can narratives broadcasted on radio find a rebirth in the digital age ? Communication au colloque Radio Content in the Digital Age, ECREALimassol Technolology University.

Barthes, R. (1981). Introduction à l'analyse structurale des récits. Paris : Seuil.

Brau, J.-L., \& Lavergne, G. (1991). La focalisation : actes du colloque international sur la focalisation narrative, Nice, 12-13-14 juin 1991. Nice : Université de Nice, Centre de narratologie appliquée.

Baudou, J. (1997). Radio mystères : le théâtre radiophonique policier, fantastique et science-fiction. Paris : INA.

Cashman, S. (1989). America in the Twenties and Thirties. New York : NY University Press.

Crook, T. (1999). Radio drama: theory and practice. London : Routledge.

Charaudeau, P. (1984). Aspects du discours radiophonique. Paris : Didier érudition.

Flynn, J. (2005). War of the Worlds : from Wells to Spielberg. Owings Mills, MD. : Galactic Books.

Lits, M. (2008). Récit et société. Louvain-la-Neuve : De Boeck.

Marion, P. (1997). Narratologie médiatique et médiagenie des récits. Recherches en communication, 7.

Osenton, T. (2002). Customer share marketing. New Jersey : Prentice Hall.

Prot, R. (2007). Précis d'histoire de la radio et de la télévision. Paris : L'Harmattan. 